\title{
Betting Patterns for Sports and Races: A Longitudinal Analysis of Online Wagering in Australia
}

\author{
Sally M. Gainsbury • Alex Russell
}

Published online: 29 October 2013

(C) Springer Science+Business Media New York 2013

\begin{abstract}
Online wagering is increasing in popularity as it is easily accessible through websites which market these services widely. However, few studies have examined online betting based on actual behavioural data. This paper describes the results of an analysis of 2,522,299 bets placed with an Australian online wagering operator over a 1-year period. The majority of bets placed were for a win $(45.31 \%)$ and were placed on races $(86.74 \%)$ or sports $(11.29 \%)$. Sports betting was dominated by ball sports, reflecting popular interest in these events. More than three-quarters $(77.63 \%)$ of the bets were losses and there was large variation in bet size between bet types and events bet on although average bets were higher than in previously reported studies. The most popular bets placed to win, had a relatively high rate of losses and lowest average returns, which may reflect less sophisticated betting behaviour. More specific handicap and total bets were placed by fewer customers, but were larger bets with the greatest returns. Similarly, bets placed on less popular sporting events had greater average returns potentially reflecting greater customer sophistication and knowledge raising the possibility of a proportion of bettors being more 'skilled'. As the first paper to analyze the types of bets placed on events and outcomes the results support the notion that wagering is an entertainment activity, and the majority of customers are motivated by factors other than simply winning money.
\end{abstract}

Keywords Betting · Wagering - Gambling $\cdot$ Sports $\cdot$ Races $\cdot$ Internet

\footnotetext{
S. M. Gainsbury ( $\square)$

Centre for Gambling Education and Research, Southern Cross University,

P.O. Box 157, Lismore, NSW 2480, Australia

e-mail: sally.gainsbury@scu.edu.au

A. Russell

School of Psychology, University of Sydney, Brennan MacCallum Building (A18),

Sydney, NSW 2006, Australia

e-mail: alex.russell@sydney.edu.au
} 


\section{Introduction}

Wagering represents approximately $5 \%$ of the global gambling market, however, betting/ wagering accounts for $43 \%$ of the online gambling market's gross revenue, predicted to reach AUD $\$ 33.7$ billion in 2012 (H2 Gambling Capital 2012). Online gambling, including wagering, is growing at an estimated $12 \%$ annually (Global Betting and Gaming Consultants 2011), driven by increased access to cheap, high-speed broadband, liberalisation of international markets, use of Internet-enabled mobile phones and wireless devices, and marketing from gambling operators (Gainsbury 2012). Wagering operators have particularly targeted sports and racing fans as potential customers and advertising and marketing associated with both major and minor teams and events is now highly prominent in markets where this is permitted (Lamont et al. 2011). Marketing efforts have increased awareness of how and where to place bets online, and, coupled with offers of free bets and bonus credits for signing-up, making deposits, and referring friends, these strategies have contributed in the growth in participation and expenditure with online wagering operators. However, ultimately the convenience and easy accessibility of online wagering is reportedly the key driver in customer uptake and participation in this mode of betting (Gainsbury et al. 2012b).

The rapid increase in online betting has caused some unease among stakeholders, including governments, sporting organisations, and community groups (Gainsbury and Wood 2011). Central concerns focus on the impact of the availability and accessibility of Internet gambling on vulnerable populations, including young people and problem gamblers, the prevention and detection of fraud and money laundering through online websites, and the ability to appropriately regulate an online activity across border states (Department of Broadband, Communication and the Digital Economy 2013; European Commission 2012; Gainsbury 2012). Further concerns have also been raised regarding the importance of safe-guarding the integrity of sports and preventing match-fixing and corruption (Department of Broadband, Communication and the Digital Economy 2013; European Commission 2012; Gainsbury 2012). However, little is known about online betting behaviour, which makes it difficult to create appropriate policies and practices to regulate this activity. The prohibition of certain types of wagering or placement of strict restrictions and requirements on wagering operators may have the unintended effect of stimulating illegal gambling, including offshore gambling sites. For example, although sports betting is illegal in the vast majority of the US, research estimates that over two thirds of Americans have gambled at least once and that over $\$ 380$ billion is wagered illegally each year (Church-Sanders 2012; Strumpf 2003. The difficulty in successfully prohibiting offshore gambling and the importance of providing a safe, regulated environment for gamblers has been cited by the majority of international governments that have liberalised online gambling (Gainsbury and Wood 2011).

Further efforts are required to examine the use of online wagering sites to determine if restrictions are warranted and to guide appropriate policy, including consumer protection and responsible gambling requirements. A greater understanding of online betting and how players typically engage in this activity would assist in guiding policy development (European Commission 2012). The current study aims to provide insight into online wagering by conducting a longitudinal analysis of actual player behaviour with an Australian online bookmaker.

Australians are among the most prolific gamblers in the world, with estimates forecasting total gambling expenditure will increase 3.3\% in 2011-2012 to reach a total of AUD $\$ 22.5$ billion (IbisWorld 2012). A recent prevalence study estimated that $64 \%$ of 
Australian adults gambled at least once in 2011, $22 \%$ bet on races and $13 \%$ bet on sports, which has doubled in popularity since the previous national prevalence survey in 1999 (Gainsbury et al. in press). The wagering market is dominated by race betting, which accounts for AUD $\$ 20$ billion in turnover, as compared to AUD\$3.3 billion for sports wagering (Deloitte 2012). Expenditure on race wagering has been fairly stable over the last 20 years and estimates indicate that racing accounts for $12.5 \%$ (AUD\$2.8 billion) of Australia's gambling expenditure (IbisWorld 2012). Sports betting expenditure for 2011-2012 was estimated at AUD\$360.5 million, representing only $1.6 \%$ of total gambling expenditure, however, this was by far the fastest growing segment of the industry, with compound annual growth of $14.7 \%$ over the past 5 years (IbisWorld 2012). Online corporate bookmakers in particular have experienced increase market share since their establishment in the mid-1990s, with compound annual growth of $9 \%$ between 2004 and 2011 compared to approximately $4 \%$ across TAB organisations (Howard 2011). These figures are consistent with reports from major wagering operators indicating that Internet wagering revenue is increasing more rapidly than retail and telephone betting. In the third quarter of the 2012 financial year, wagering provider Tabcorp reported an increase of $18.0 \%$ on its Internet wagering turnover in the year to date as compared to a retail growth of $0.9-7.0 \%$, and a decline in phone and oncourse betting (Tabcorp 2012).

Internet gambling is prohibited in Australia, with the exception of online wagering and lottery provided by licensed operators. Participation in online gambling is relatively low, with $8 \%$ of Australian adults reporting having gambled online in 2011, although this represents a sizeable increase from approximately $1 \%$ estimated to gamble online in 1999 (Gainsbury et al. in press). In a recent survey of 6,682 Australian gamblers, including 4,680 (70 \%) Internet gamblers, betting at land-based venues accounted for only $30 \%$ of sports betting and $33 \%$ of race wagering, with computer and mobile-based online gambling representing the most common method of placing bets (Gainsbury et al. 2012a, b). The most common advantages cited by players to explain the use of Internet gambling included the lack of travel needed, convenience and accessibility, the lack of crowds and unpleasant people, greater privacy and anonymity and greater physical comfort. Land-based gambling being unavailable was cited by only $2 \%$ of Internet gamblers suggesting that lack of access to these traditional forms is not the reason behind the shift to Internet betting. These results indicate that although they could bet in venues or via the telephone, an increasing proportion of bettors prefer to use the Internet.

Previous research has had to rely on inaccurate self-reports of betting behaviour but the use of data from player account-based gambling has begun to make a substantial contribution to the understanding of gambling behaviour and player profiles (Gainsbury 2011). Analysis of the betting patterns of 40,499 Internet sports gamblers that subscribed to a European wagering site in 2005 provides an insight into the typical behavioural patterns of these relatively early adopters of online gambling (LaBrie et al. 2007). Over an 8-month period the vast majority of fixed odds sports bettors appeared to bet in a relatively moderate fashion, placing an average of 2.5 bets of $€ 4$ every fourth day and losing $29 \%$ of the amount wagered (LaBrie et al. 2007). A small proportion of bettors appear to be highly involved in terms of the amounts wagered or number of bets placed. The top $1 \%$ of the sample in terms of amount wagered had lower percent losses than the rest of the sample, which the authors interpreted as meaning that winning may encourage further betting (LaBrie et al. 2007). An alternate explanation is that a small proportion of online bettors are more sophisticated and use resources and strategies to reduce their losses, who may also receive favourable odds and rebates from operators due to the size of their wagers. The players studied were most likely to be young German men and given that the wagering 
analysed was done in 2005 the extent to which the results can be applied to other populations today is unclear. Furthermore, although fixed-odds and live-action bettors were compared, no analysis was conducted based on the types of bets made or events bet on, which may provide useful insights about player behaviour.

A more recent analysis of player account data analysed the wagering activity of 11,394 customers of a large Australian totalisator wagering operator over a 10-year period, from 2001 to 2010 (Gainsbury et al. 2012a). On average, players were active for a period of 5.8 years during this time, bet on 46 days and made 718 bets over the life of their account. The majority of players (92\%) lost money overall and across all accounts on average players lost $34 \%$ of the total amount wagered. Most players appeared to bet moderate amounts, with average bet sizes ranging from $\$ 14.05$ to $\$ 198.82$. However, similar to the results of La Brie and colleagues (2007), these results were impacted by a small minority of bettors who accounted for the majority of bets (top $1 \%$ of all bets placed), but had the lowest proportional net losses (an average loss of $20 \%$ of the total amount they wagered). Although these results only represent bets placed with one operator, they provide valuable insight into typical betting patterns. These results are similar to figures released by a large totalisator Tabcorp which reported average bet sizes of AUD\$11.35 in New South Wales (NSW) and \$12.05 in Victoria (VIC; Masters 2011). Win bets were reported to be highly popular, representing over half of all turnover in NSW and $42 \%$ in VIC, and 26-44\% of customers place multi-bets. However, without a detailed analysis of all player behaviour it is difficult to put these figures in context.

The current study aimed to analyse player account data from an Australian corporate bookmaker. Investigation of online wagering patterns will further the understanding of how individuals wager online, including the types of bets placed and the outcomes of these. This information can be used by stakeholders, including policy makers, who may be able to implement a more targeted approach relevant to this type of gambling. Given the few studies conducted examining online wagering, and that this is the first study to examine player account data from a corporate bookmaker, no specific hypotheses were formed. This study aimed to provide a descriptive outline of the types of bets made and the outcomes of bets and compare wins and losses and gambling outlay and returns. These variables were chosen as the focus of this study as they were available in the dataset and have been examined in previous populations of online bettors so will contribute and extend the extant literature on this topic.

\section{Methods}

Sample

An Australian corporate bookmaker, Betchoice (now trading under the name Unibet) provided a database of de-identified account information for research purposes. Betchoice was formed in 2001 and licensed in the Northern Territory to provide online wagering in addition to telephone betting in 2006. The principle focus of Betchoice was providing bets on races as opposed to sports, although a wide variety of markets are available for customers. Although approximately 40,000 customers have accounts with Betchoice, many of these are not active clients, defined as clients that place at bet at least once every 12 months. Bookmakers offer fixed-odds bets, the odds may change during the betting period, but the odds for each bet is determined at the time the bet is placed. 
The dataset contains bets and transactions placed online through the Betchoice website and via telephone from January 1, 2010 until December 31, 2010. In total there were $3,517,480$ lines of information, including 587,107 payouts, 198,197 money transfers into and out of accounts, 181,564 bets between bookmakers, 21,618 cancelled, 6,280 bets unfinalised, 400 rejected bets and 15 accepted bets that had not been completed. When these were removed, there were 2,522,299 completed bets from 12,099 different user accounts. The vast majority $(96.21 \%)$ of bets were placed via the Internet, and the remaining $3.79 \%$ were placed by telephone.

\section{Variables}

The dataset included a unique user identification number for each bet. No demographic data were available, so all analyses were conducted based on bets placed. Two variables were included in the dataset that recorded the size of the bet. One recorded the amount of money placed by the bettor (bet outlay), while the second recorded the overall size of the bet, including any bonuses. For example, a $\$ 5$ free bet would have $\$ 0$ wagered by the bettor (bet outlay), but have an effective total bet size of $\$ 5$. In most cases, summary statistics of bet outlay and total bet size were within $\$ 1.00$ of each other, so only bet outlay is reported below. Where larger differences exist, this has been noted under Tables 1 and 2 . Where an analysis is reported below for bet outlay, the same analysis was tested for total bet size. The pattern of results was the same for bet outlay and total bet size for all analyses.

Table 1 Description of each variable in the dataset

\begin{tabular}{ll}
\hline Bet type & Description \\
\hline UserID & A numerical code for each user account \\
TransactionID & A unique numerical code for each transaction line \\
EventID & A numerical code for each event (e.g., a match, race, etc.,) \\
Transaction date & Date on which the transaction was placed \\
Transaction time & The time when the transaction was placed \\
Sport & Sport on which the bet was placed, although could also indicate events other than \\
& sports, such as races and elections \\
Event & Full event name (e.g., Ipswich Race 9-Merry Christmas CI2 Hcp) \\
Bet type & Type of bet (e.g., win, each way). See Table 3 \\
Outcome & The outcome on which the bet was placed (e.g., Dallas Mavericks 1st-10) \\
Bet amount & Amount that the user placed on the bet (referred to as bet outlay) \\
Total amount & Amount that the user placed on the bet plus any additions, such as free bets \\
Dividend & Amount that the user won as a result of the bet, not including the initial stake \\
Bet status & Whether the bet was a loss (coded as no Return), win (Return @ transactionID) or \\
Balance effect & various other things, such as cancelled, transfer, etc \\
ReturnID & The same as total amount above, but recorded as a negative value \\
Bet method & In the case of a winning bit, this links the bet line to the payout line in the data \\
Stat result & Either internet or telephone \\
\hline & The amount won or lost
\end{tabular}




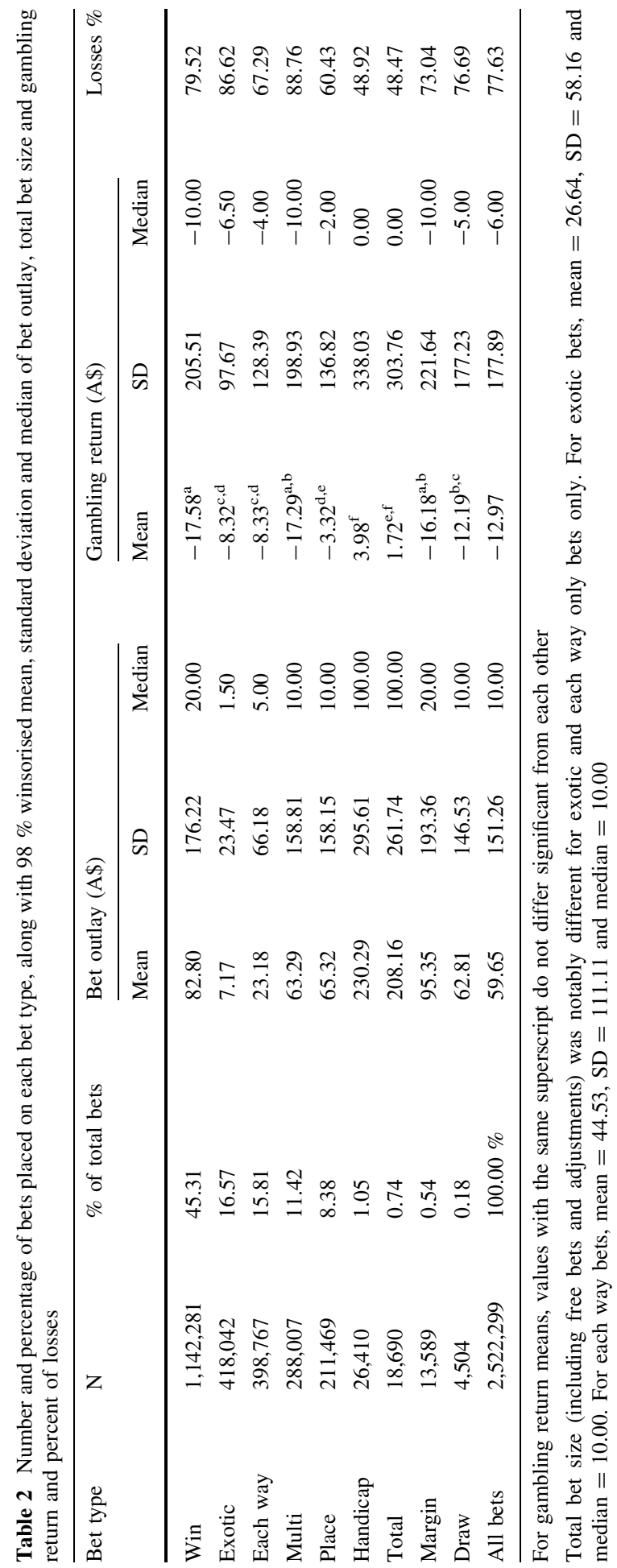


All bets were recoded as either a win or a loss, based on the bet status variable. All bets that were classified as "No return" were considered to be losses, while those with a Return (payout) line were considered wins. Wagering return was recorded by a separate variable, where a loss is recorded as negative total bet size, while a win is recorded as whatever the bettor won above their total bet size. So, if a bettor placed a total bet (including any bonuses) of $\$ 10$ on an outcome paying $\$ 1.50$ and lost, the gambling return would be $-\$ 10$, whereas if the bettor won, the gambling return would be recorded as $\$ 5$. In some cases, despite the bet being recorded as a win, the gambling return was negative. For example, in one case, the bettor placed a $\$ 500$ each way bet, for a gambling return of $-\$ 290$. In this case, the horse would have placed without winning, so the bettor received $\$ 210$ of their bet back, for a loss of $\$ 290$.

The winning amount for multi bets was not present in the dataset and was estimated by multiplying the total bet outlay by the recorded dividend and subtracting the total bet outlay from the product. When the same thing was done for other bet types, the estimated win correlated perfectly or highly $(r>0.96)$ with the recorded win, with the exception of place bets $(\mathrm{r}=0.69)$, each way bets $(r=0.51)$ and exotics (bets other than the traditional win/place, e.g., Quinellas, Trifectas, Exactas and Quadrellas), $r=0.08$ ), thus verifying this procedure.

To avoid undue influence of extreme values, bet outlay, total bet and gambling return variables were winsorised so that values beyond the 1st and 99th percentile were recoded as having the same value as that percentile. However, when minimums and maximums are reported, this refers to unwinsorised data. A description of the relevant variables included in the dataset is provided in Table 1.

Analysis

Due to the extremely large number of bets being analysed, almost all inferential statistical results were significant at an alpha of 0.001 . Homogeneity assumptions were violated for the ANOVAs and thus the more robust Brown-Forsythe test is reported below.

\section{Results}

Descriptives

Types of Bets

As shown in Table 2, $45.31 \%$ of all bets were placed for a win, with $16.57 \%$ on exotics $15.81 \%$ placed on an each way bet, $11.42 \%$ were multi-bets, and $8.38 \%$ were for a place. All other bet types, including handicap, total, margin, and draw, and together represented less than $3 \%$ of all bets placed. Multi-bets were treated as a single bet and distinct from the other categories, even though there may have been some cross-over, for example with a multi-bet that consisted of legs that were all placed for a win. For a description of the different bet types, see Table 3 . 
Table 3 Description of different bet types

\begin{tabular}{ll}
\hline Bet type & Description \\
\hline $\begin{array}{l}\text { Win } \\
\text { Exotic }\end{array}$ & A bet placed on who will win the race, match or contest \\
Bets where a particular series of outcomes is required, such as a Trifecta, where the \\
aim is to pick the first three horses. Includes Exacta, Quinella, Trifecta, Any 2, \\
Pick 4 or First 4 and others \\
Part of the bet is placed on a win and part on the horse coming in a place \\
Each way \\
$\begin{array}{l}\text { A bet made up of multiple "legs", where each leg is a bet. All bets must win in } \\
\text { order for the multi to win }\end{array}$ \\
A bet placed on whether the horse finishes in the top three places (or in some cases, \\
the top two places) \\
One team or player is given an advantage, so that even if the other team or player \\
wandicap \\
A bet they have to win by a certain amount for this bet to be successful \\
$\begin{array}{l}\text { Total } \\
\text { Margin }\end{array}$ \\
$\begin{array}{l}\text { Where the aim of the best is to pick the winning margin (e.g., 1-12 points) } \\
\text { A bet on neither team or player winning }\end{array}$
\end{tabular}

\section{Events Bet On}

The vast majority of bets $(86.74 \%)$ were placed on races. There is no distinction in the data file between different types of horse racing (harness, trots, etc.,), nor is there a category for dog racing, so this figure most likely includes all of these forms. The second highest category was sports betting $(11.29 \%)$, in particular ball sports (11.00\% of all bets placed). Thus, the vast majority $(97.39 \%)$ of sports bets were placed on ball sports, in particular on the various codes of football (54.98\% of sports bets). When all forms of football were combined (soccer, Rugby Union, Rugby League, Australian Rules, American football), they accounted for $6.21 \%$ of all bets placed, compared to non-football ball sports (e.g., cricket, netball, basketball, baseball, etc.,), which accounted for $4.79 \%$ of all bets. Football bets were largely accounted for my soccer, rugby league and Australian Rules and bets on non-football ball sports were most likely to be on basketball or tennis.

In addition to the single sports bets, $1.94 \%$ were classified as mixed bets. These were all multi-bets and could thus include bets on a combination of horse racing, sports betting, and other categories, although the majority $(>90 \%)$ of mixed bets were combinations of ball sports, in particular football and basketball. Only 774 bets $(0.03 \%)$ were placed on novelty events, such as elections, entertainment, and social occurrences (see Table 4).

\section{Win and Loss Comparisons}

As shown in Table 1, more than three quarters (77.63\%) of all bets placed were losses. The types of bets with the highest losing percent of bets were multis $(88.76 \%)$, exotics $(86.62 \%)$ and wins $(79.52 \%)$. Slightly more than half of both handicap and total bets were wins.

When the six different categories (racing, football, ball sports other than football, nonball sports, mixed and other) were compared, all groups were significantly different to each other, except for football and non-ball sports, $\chi^{2}(5, N=2522299)=19,527.08$, $p<0.001, \phi=0.09$. Mixed bets were the least successful ( $85.70 \%$ losses), followed by bets on horse racing $(78.60 \%)$, football and non-ball sports $(72.78$ and $71.69 \%$ 


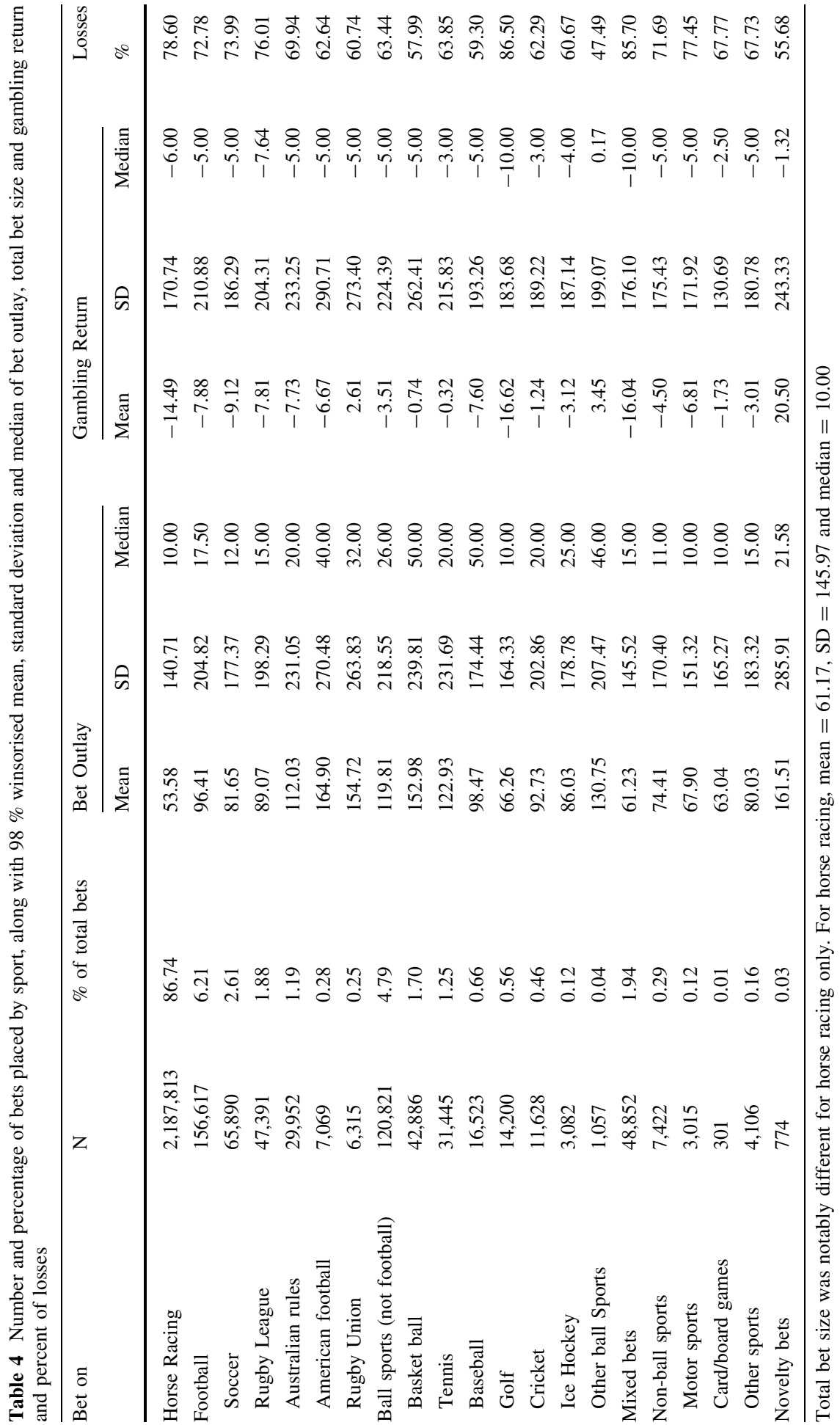


respectively), ball sports other than football (63.44\%), and novelty bets (55.68 \%). This latter category consists mostly of election and social bets.

\section{Gambling Outlay and Return by Various Gambling Types}

Minimum bets for all forms of gambling were $\$ 1$, with the exception of exotics and wins, where bets less than $\$ 1$ are allowed. The largest bets placed were eight $\$ 100,000$ bets, all from the same bettor, all on racing and consisted of one multi bet and seven bets for a win. Apart from racing, the next largest bet placed by sport was $\$ 80,000$ (rugby league), $\$ 70,000$ (Australian Rules), $\$ 50,000$ (rugby union) and $\$ 46,000$ on a soccer World Cup bet. Of the 2,522,299 bets placed, $423(0.02 \%)$ were $\$ 10,000$ or more, 35,722 bets $(1.42 \%)$ were $\$ 1,000$ or greater and there were 403,796 bets $(16.00 \%)$ placed for $\$ 100$ or more.

In terms of winsorised bet outlay, all bet types were significantly different from each other except for draw, multis and place, Brown-Forsythe $F(8,105716.3)=12,536.40$, $p<0.001, \eta^{2}=0.06$. The greatest bets were the more specialised handicap and total bets, and although these also had high levels of variation, the medians were still significantly higher than other bet types. Bettors also wagered greater amounts on win and margin bets. Statistically significant differences were also found between the various bet types in terms of gambling return, Brown-Forsythe $F(8,109419.7)=206.24, p<0.001, \eta^{2}<0.01$. The greatest returns were for handicap and total bets, followed by place bets. However, the pairwise differences here are slightly more complex and are indicated by subscripts in Table 1 .

When comparing the six categories (racing, football, ball sports other than football, nonball sports, mixed and other) in terms of mean winsorised bet outlay, all were significantly different from each other except for racing and mixed bets, Brown-Forsythe $F(5$, $5451.9)=3,377.76, p<0.001, \eta^{2}=0.01$. The greatest bets were placed on novelty and non-football ball sports, followed by football, non-ball sports, and then mixed bets and racing. Median bets are in a slightly different order, demonstrating the variance in results. Within non-football ball sports, the greatest best were placed on basketball, other ball sports, and tennis and within football, the greatest bets were placed on American Football, rugby union and Australian Rules.

In terms of gambling return, novelty bets were significantly higher than all other bet types, whereas racing was significantly lower than non-ball sports and ball sports other than football. Mixed bets and football bets were not significantly different to each other or racing, non-ball sports and ball sports other than football, Brown-Forsythe $F(5$, $10717.1)=153.01, p<0.001, \eta^{2}<0.01$. Within non-football ball sports, other ball sports, tennis and basketball had the greatest returns and within football, rugby union bets had the greatest return.

\section{Discussion}

Consistent with the aim of this paper, the betting patterns of gamblers using an online bookmaker have been described, based on a database of all bets placed in one calendar year. Approximately half of all bets were placed for a win, which is similar to the results reported by another Australian betting operator, Tabcorp (Masters 2011). This may indicate that bettors prefer simple, uncomplicated bets, and are more comfortable identifying just one potential winner. This may also be considered a bolder betting style, as opposed to 
bets each way, which reflect a more conservative, cautious, and risk-averse approach. Place bets were more successful than win bets, both in terms of percentage of bets that win and in gambling return, although the latter could simply be due to larger bets being placed for wins. That a higher proportion of place bets were successful compared to wins may not be very surprising, given than there are more possible successful outcomes for place bets than for win bets. Exotic bets and multi bets are relatively risky, as they require several predictions to be fulfilled, although, as a result they generally give a larger return if successful. As risk preferences and motivations for placing types of bets were not measured these hypotheses should be explored in subsequent research. Given that the operator is predominantly a race betting specialist, it was not surprising that the vast majority of bets were on races. This is consistent with the Australian wagering market, which is dominated by race wagering, despite the rising popularity of sports betting.

Sports betting was dominated by ball sports and various codes of football, in particular soccer, rugby league and Australian Rules. The FIFA World Cup was held in 2010, which is likely to explain the large proportion of soccer bets. Industry reports estimated that AUD \$4.9 billion was wagered globally on this tournament, including AUD \$110 million in Australia, which is a huge increase on the estimated AUD\$1.1 billion spend on the previous tournament (Church-Sanders 2012). A report on the UK online gambling market stated football (soccer) betting has seen the largest growth in the sports betting sector, growing $69 \%$ between 2009 and 2012 (Charlton 2013). However, this has largely been driven by the popularity of in-play betting, which is not permitted on regulated Australian wagering sites.

In addition to soccer bets, substantial betting on rugby league and Australian rules is consistent with estimates from other Australian online wagering operators that suggest these sports account for over $50 \%$ of all sports betting in Australia, and $7 \%$ of all wagering (Deloitte 2012). The findings also likely reflect the greater consumer interest in and popularity of various sporting events. According to the Australian Bureau of Statistics, $43 \%$ of Australians aged 15 years and over attend at least one sporting event per year and the most popular sports are Australian Rules football, horse racing, rugby league, motor sports, and soccer (Australian Bureau of Statistics 2010). Slightly different results are found for watching sports on television, which is led by cricket, Australian Rules football, tennis, rugby league and the Melbourne Cup (Roy Morgan 2012). The betting patterns are consistent with US research which suggests that betting trends follow popular sports spectatorship and bettors, like fans, appear to enjoy seeing the best teams and preferring games televised on major networks (Paul and Weinback 2013, 2010). The bookmaker analysed in the current study was not heavily advertising before or during the period analysed, so these results are unlikely to be influenced by marketing tied to sporting events. It is possible that these findings support the notion that betting and watching sporting events are complimentary activities for most bettors, as opposed to betting being an independent activity related to maximising profitable returns (Paul and Weinback 2013). However, as motivations for betting were not directly measured in the current research these hypothese require investigation in future studies.

Overall, the majority of bets resulted in losses, which expected based on previous studies of wagering (Gainsbury et al. in press; LaBrie et al. 2007; Paul and Weinback 2013). Online bookmakers have reported increasing revenue over the past few years, which is generated from an increasing customer base, and a combination of lost bets and odds ensure that bookmakers take a margin (commission) of bets placed (Raventós and Zloezzi 2011). The results of this study may imply that the bookmaker studied is appropriately skilled in predicting the outcomes of events and exploiting this advantage by strategically 
setting odds that ensure that the majority of bets are lost, or at least return an affordable proportion of wagers to customers so that they continue betting over time, but are unlikely to make a profit. This is consistent with previous research showing that the market for sports gambling is structured to allow bookmakers to achieve substantially high profits (Levitt 2004). The average bet size (\$7.17-230.16) was considerably larger than that reported by LaBrie and colleagues $(€ 4 ; 2007)$ and by Gainsbury et al. $(\$ 14.05-\$ 18.82$; 2012a). Furthermore, many large bets were placed, including risky multi bets, although the majority of bets were for reasonable amounts, depending on how many bets each customer made over a period of time. This may reflect a tendency for customers to place larger bets with an online bookmaker than a totaliser agency. Given the complexity of this topic, an analysis of individual bettor behaviour is the subject of another paper.

The most popular type of bet, to win, had a relatively high rate of losses and the lowest average gambling returns, which may be explained by the predominance of racing bets and the greater difficulty in picking one winner in a field with multiple contenders. Alternatively, these bets may be more likely to be placed by less sophisticated customers who have a lower ability to accurately assess the relevant factors and pick the best event and winner to successfully back. For example, bettors have been shown to have a bias to bet on longshots in pari-mutuel horse-race betting (Golec and Tamarkin 1998; Jullien and Salanie 2000; Vaughn Williams and Paton 1997), which may also be true for fixed-odds betting. Similarly, bettors have been shown to bet a disproportionate amount on home teams and underdogs (Golec and Tamarkin 1991; Gray and Gray 1997; Paul and Weinbach 2007, 2008). If odds are balanced, bettors should place bets evenly on each side or outcome, therefore, the outcomes of previous research may imply that bettors may be influenced by emotions and preferences when placing bets, rather than probability of economic outcomes. Furthermore, Levitt (2004) found that sportsbooks can consciously exploit the desire of bettors to back the favourite team by giving bettors on that team poorer odds. This may further explain why the majority of bets result in losses, if bettors are choosing wagers based on preferences for a team to win, rather than the likelihood of winning the bet (Raventós and Zloezzi 2011). The cognitive processes and strategies used by customers in selecting bets should be examined in future research.

Multi-bets represented a significant portion of bets, but customers tended to bet lower amounts than for wins and places, perhaps suggesting that they may be less confident in the outcome. This strategy may be appropriate given that multi-bets resulted in the highest proportion of losses. The lowest average bets were for exotic bets, which also had a relatively low standard deviation, indicating that most customers wagered small amounts on these bets, which are based on very specific outcomes. Again, unsurprisingly, the vast majority of exotic bets resulted in losses, suggesting that low bets are appropriate, although the returns for these bets were more favourable than for wins, as these bets typically have good payout rates if successful. The placement of small bets on riskier activities is consistent with Conlisk's (1993) "Small Gamble Theorem", suggesting that individuals obtain utility from gambling on small bets as a risk-aversion strategy when the stakes are high. Lower amounts were generally placed on each way bets, which may be due to the lower odds typically offered for these bets, so players do not have the same opportunity to win large payouts. Correspondingly, each way bets had a lower proportion of losses, likely related to the greater number of outcomes to result in a winning bet. Each way bets may be more popular with risk-averse bettors, who would be happy with small outcomes, but reduced losses. Alternatively, consistent with Conlisk's (1993) Theorem, each way bets may be placed by bettors as a form of insurance against the riskier bets placed, in the hopes of winning some of their bets. This perceived insurance may lead some bettors to place an 
each way bet instead of taking the risk of either a win (smaller chance of a higher return) or place (higher chance of a smaller return) and thus engaging in a bet when they would not do so if the each way option were not available.

Of interest, the more specific handicap and total bets were placed by few customers, but these represented the largest average bets and the greatest return to players, although there was also large variation in these results. On average, half of the place and handicap bets made resulted in wins, in contrast to all other types of wagers. These results are quite significant as they suggest that it is possible for bettors to come out ahead. Customers who made these types of bets may have greater knowledge and understanding of specific races and the factors that affect outcomes and subsequently be more successful with their bets. Alternatively, they may be more astute in finding bets with favourable odds. These results are consistent with the findings from an analysis of another Australian wagering operator, which found that a small proportion of bettors were able to minimise their losses (Gainsbury et al. 2012a). This study did not investigate the type of bets made by the more successful bettors, nor did the work by La Brie and colleagues (2007). There is evidence of subgroups in the heterogeneous population of bettors, including demonstrated differences in betting behaviour and performance between recreational and professional bettors (Bruce et al. 2012; Gainsbury et al. 2012a) These findings suggests that further research is needed to examine this subgroup of potentially successful bettors and factors related to bets resulting in a win.

Bets placed on races tended to be smaller on average compared to other events. However, those betting on races tended to lose more money per bet, on average, than all other forms of betting apart from mixed bets. This may reflect the interest in race betting as an entertainment activity rather than an expectation of consistent winning or positive returns. In relation to sports betting, bet outlays and outcomes differed between the types of event bet on, indicating that customers had different betting strategies for different events. Mixed bets placed on multiple sporting events were the least successful, which is not surprising as a greater number of successful predictions are required to win. This may explain the relatively few bets placed on these events and lower bet outlay compared to other sports. Football bets were on average significantly smaller and less successful than bets placed on non-football ball sports. Soccer, Rugby League, and Australian Rules games appear to be particularly difficult to predict, judging from the lower returns. In contrast, bets on Rugby Union, had a positive return on average, although over half still resulted in losses, perhaps suggesting that some astute customers were able to identify more favourable odds for rugby union matches and subsequently won large amounts, impacting the overall average results. An alternative explanation may be that events in which outcomes are more difficult to pick have less favourable odds, given no clear likely winner, resulting in a lower proportion of returns. Given the absence of prior studies examining types of bets made on sports events and the outcomes of these, including the motivations of bettors, interpretation of these results requires some caution until further research can be conducted.

Similar to the outcome of more specialised racing bet types, bets placed on less popular events, i.e., other ball sports, were the most successful. Wagers placed on basketball, baseball, and ice hockey had a lower proportion of losses than football bets. Similarly, tennis, basketball, cricket and ice hockey bets had the greatest average returns (not including rugby union and other ball sports), although these were still losses on average. However, there was also relatively high variability in results indicating that the outcomes of bets on these events are not consistent. Because betting on these events is less popular, these events may be subject to less scrutiny and research by the bookmaker. This may 
assist some customers who are more sophisticated bettors and have greater knowledge or ability to find favourable odds as compared to the majority of customers who may give less consideration to their bets (Raventós and Zloezzi 2011). These are also all sports in which a few 'star' players may influence the outcome of a game through point scoring, or skilled defence, perhaps making judgments on outcomes more reliable. However, the notion of skilled betting has been contested by some researchers who contend that so-called "skills" of sports and race bettors are cognitive distortions and other factors explain more successful outcomes (Cantinotti et al. 2004; Ladouceur et al. 1998).

Novelty events include bets placed on the outcome of elections and entertainment or popular events, such as who will win the latest reality television show. Few bets were placed on these events, which is unsurprising as these are generally intended to be nonserious bets that are provided for interest and novelty value. However, novelty bets had the highest returns. This may reflect a tendency for the operator to be willing to lose some money on these types of bets, which are primarily designed to generate interest and discussion, as opposed to contributing to profits. For example, online operator Sportsbet paid out both ways on a novelty bet offered on whether the ratings of a reality show would increase or decrease due to ambiguous ratings data, so all punters won and the operator received free publicity, for only $\$ 11,000$ (Ellis 2012).

\section{Limitations}

As the dataset included all bets placed over an entire year, the results are relatively robust in terms of capturing seasonal variation in betting patterns. However, it is important to note that betting patterns are related to major sporting events and 2010 was slightly unusual in having two AFL grand finals, as well as a Soccer World Cup. The dataset only accounts for betting with one operator, which specialises in race wagering, so the results may not be representative of online wagering on sports with other operators more generally. Bets placed with other operators are obviously not accounted for, nor is other gambling involvement, limiting the description of overall betting patterns. Furthermore, despite the richness of information obtained from actual behavioural data, no contextual data or personal variables were included so it is not possible to understand the customers beyond their behaviour. Future studies should attempt to combine self-reported data, for example, other gambling behaviour, personal characteristics, thoughts and attitudes, and risk for problem gambling to provide a more holistic account of online bettors (Gainsbury 2011).

\section{Conclusions}

Notwithstanding the limitations, the strength of this analysis is the longitudinal analysis of an entire sample of active bettors, which accurately describes all bets made during the period examined. This represents a significant contribution to the field as it is the first paper to specifically examine the types of bets placed on events and outcomes. It enables for the first time, a detailed understanding of how players place bets and the typical outcomes. The results of these analyses show that overall, although the majority of bets place result in losses, these losses were relatively moderate. Wagering operators tend to return a larger proportion of bets to customers, unlike casino games and slots, which tend to offer less favourable player returns (Church-Sanders 2012). Further research is required to specifically compare betting patterns and outcomes for wagering and gaming, which is important to determine whether different policies and regulations should be applied to these activities. 
After race wagering, which was the predominate activity of the online wagering operator examined, bettors appear to be most likely to place bets on popular sporting events, suggesting that this activity is an adjunct to watching sports and races. As the majority of bets resulted in losses, this suggests that sports and race wagering may not be an activity in which the customers aim to make money, as otherwise they would cease betting in the face of continual losses. Rather, it supports the notion that, for at least some customers, wagering is an entertainment activity, motivated by factors other than simply winning money. Further research is required to examine the individual factors and motivations for betting. Ideally, any research in which contextual details about individual bettors could be linked to player-account data as this would provide an extremely useful insight into how motivation affects actual gambling behaviour. Further research may also identify specific betting patterns and subgroups of players. This may lead to the development of different consumer protection and responsible gambling strategies for players based on their individual gambling patterns (Gainsbury 2011). Optimally, combining selfreport with analysis of actual player behaviour may provide practical insights into betting behaviour for key stakeholders, including policy makers, and lead to the development of appropriate regulation that does not overly restrict recreational players, but offers appropriate safeguards.

Acknowledgments The authors would like to acknowledge and thank the European Association for the Study of Gambling who provided funding for this research for the first author as well express their appreciation to Unibet and Mr. Mark Morrissey who provided the data analysed in this paper.

\section{References}

Australian Bureau of Statistics. (2010). 4174.0 — spectator attendance at sporting events, 2009-2010. Australian Bureau of Statistics, retrieved from http://www.abs.gov.au/ausstats/abs@.nsf/Products/ 4174.0 2009-10 Main+Features Most+popular+sports+attended?OpenDocument.

Bruce, A. C., Johnson, J. E. V., \& Peirson, J. (2012). Recreational versus professional bettors: Performance differences and efficiency implications. Economics Letters, 114, 172-174.

Cantinotti, M., Ladouceur, R., \& Jacques, C. (2004). Sports betting: Can gamblers beat randomness? Psychology of Addictive Behaviors, 18, 143-147.

Charlton, G. (2013). UK’s online gambling sector worth £2bn in 2012: stats. Econsultancy. Retrieved from http://econsultancy.com/nz/blog/62407-uk-s-online-gambling-sector-worth-2bn-in-2012-stats.

Church-Sanders, R. (2012). Digital sports betting: A market assessment and outlook (2nd ed.). London: iGaming Business.

Conlisk, J. (1993). The utility of gambling. Journal of Risk and Uncertainty, 6, 255-275.

Deloitte (2012). Optimal product fee models for Australian sporting bodies. Deloitte Touche Tohmatsu.

Department of Broadband Communications and the digital economy. (2013). Final report 2012: Review of the interactive gambling act 2001. Canberra: Department of Broadband Communications and the Digital Economy.

Ellis, S. (2012, Jul 24). So, did The Shire rate higher... or not? Sydney morning Herald. Retrieved from http://www.smh.com.au/entertainment/tv-and-radio/so-did-the-shire-rate-higher-or-not-20120724-22mn1. html.

European Commission (2012). Commission sets out action plan for online gambling. Retrieved from http:// ec.europa.eu/internal_market/services/gambling_en.htm.

Gainsbury, S. (2011). Player account-based gambling: Potentials for behaviour-based research methodologies. International Gambling Studies, 11, 153-171.

Gainsbury, S. (2012). Internet gambling: Current research findings and implications. New York: Springer.

Gainsbury, S., Russell, A., Hing, N., Wood, R., \& Blaszczynski, A. (in press). The impact of Internet gambling on gambling problems: A comparison of moderate-risk and problem Internet and nonInternet gamblers. Psychology of Addictive Behaviors. Advance online publication Feb 25, 2013. doi:10.1037/a0031475. 
Gainsbury, S, Russell, A., Hing, N., Wood, R., Lubman, D. \& Blaszczynski, A. How the Internet is changing gambling: Findings from an Australian prevalence survey. Journal of Gambling Studies. Advance online publication. doi:10.1007/s10899-013-9404-7 (in press).

Gainsbury, S., Sadeque, S., Mizerski, R., \& Blaszczynski, A. (2012a). Wagering in Australia: A retrospective behavioural analysis of betting patterns based on player account data. Journal of Gambling Business and Economics, 6(2), 50-68.

Gainsbury, S., \& Wood, R. (2011). Internet gambling policy in critical comparative perspective: The effectiveness of existing regulatory frameworks. International Gambling Studies, 11, 309-323.

Gainsbury, S., Wood, R., Russell, A., Hing, N., \& Blaszczynski, A. (2012b). A digital revolution: Comparison of demographic profiles, attitudes and gambling behaviour of Internet and non-Internet gamblers. Computers in Human Behaviour, 28, 1388-1398.

Global Betting and Gaming Consultants (2011). Global gaming report (6th ed.). Castletown, Isle of Man, British Isles: Author.

Golec, J., \& Tamarkin, M. (1991). The degree of price inefficiency in the football betting markets. Journal of Financial Economics, 30, 311-323.

Golec, J., \& Tamarkin, M. (1998). Bettors love skewness, not risk, at the horse track. Journal of Political Economy, 106, 205-225.

Gray, P., \& Gray, S. (1997). Testing market efficiency: Evidence from the NFL sports betting market. Journal of Finance, 52, 1725-1737.

H2 Gambling Capital (2012). Preliminary results 2011 H2 eGaming dataset now available. H2 Gambling Capital. Retrieved from http://www.h2gc.com/article/preliminary-results-2011-h2-egaming-datasetnow-available.

Howard, J. (2011). Australian racing fact book. Racing Information Services Australia. Retrieved from http://www.australianracingboard.com.au.

IbisWorld. (2012). Footy season kicks off the annual surge in sports betting. Melbourne, Australia: IbisWorld.

Jullien, B., \& Salanie, B. (2000). Estimating preferences under risk: The case of racetrack bettors. Journal of Political Economy, 108, 503-530.

LaBrie, R. A., LaPlante, D. A., Nelson, S. E., Schumann, A., \& Shaffer, H. J. (2007). Assessing the playing field: A prospective longitudinal study of internet sports gambling behaviour. Journal of Gambling Studies, 23, 347-362.

Ladouceur, R., Giroux, I., \& Jacques, C. (1998). Winning on the horses: How much strategy and knowledge are needed? Journal of Psychology, 132, 133-142.

Lamont, M., Hing, N., \& Gainsbury, S. (2011). Gambling on sport sponsorship: A conceptual framework for research and regulatory review. Sport Management Review, 14, 246-257.

Levitt, S. D. (2004). Why are gambling markets organized so differently from financial markets? The Economic Journal, 114, 223-246.

Masters, R. (2011, Aug 3). Brash gamblers still think inside the box. Sydney morning Herald. Retrieved from: http://www.smh.com.au/business/brash-gamblers-still-think-inside-the-box-20110802-1i9xh.html.

Paul, R., \& Weinbach, A. (2007). Does sportsbook.com set pointspreads to maximize profits? Tests of the Levitt model of sportsbook behavior. Journal of Prediction Markets, 1, 209-218.

Paul, R., \& Weinbach, A. (2008). Price setting in the NBA gambling market: Tests of the Levitt model of sportsbook behavior. International Journal of Sport Finance, 3, 2-18.

Paul, R. J., \& Weinback, A. P. (2010). The determinants of betting: Volume for sports in North America: Evidence of sports betting as consumption in the NBA and NHL. International Journal of Sports Finance, 5, 128-140.

Paul, R. J., \& Weinback, A. P. (2013). Baseball: A poor substitute for football: More evidence of sports betting as consumption. Journal of Sports Economics, 14, 115-132.

Raventós, P., \& Zloezzi, S. (2011). Sportsbooks and politicians: Place your bet! Journal of Business Research, 64, 299-305.

Roy Morgan (2012, Jan 25). Cricket and AFL dominate sports watched on TV. Roy Morgan online store. Retrieved from http://www.roymorganonlinestore.com/News/Cricket-and-AFL-dominate-sportswatched-on-TV.aspx.

Strumpf, K. S. (2003). Illegal sports bookmakers. Working paper. Department of Economics, University of North Carolina at Chapel Hill, 1-60.

Tabcorp (2012, May 3). 3Q12 Trading update. Tabcorp Holdings Ltd. Retrieved from http://www.tabcorp. com.au/investor-centre_annual-reports.aspx.

Vaughn Williams, L., \& Paton, D. (1997). Why is there a favourite-longshot bias in British racetrack betting markets? Economic Journal, 107, 150-158. 